\title{
A structured group exercise program for patients with metastatic cancer receiving chemotherapy and CTNNBI ( $\beta$-catenin) as a biomarker of exercise efficacy
}

\author{
This article was published in the following Dove Press journal: \\ Cancer Management and Research \\ 13 October 2017 \\ Number of times this article has been viewed
}

\author{
James A Chiarotto' \\ Riyad Akbarali ${ }^{2}$ \\ Lara Bellotti ${ }^{2}$ \\ George Dranitsaris ${ }^{3}$ \\ 'Department of Medicine, \\ ${ }^{2}$ Department of Cardiac \\ Rehabilitation, Scarborough and \\ Rouge Hospital, ${ }^{3}$ Augmentium Pharma \\ Consulting, Inc., Toronto, ON, Canada
}

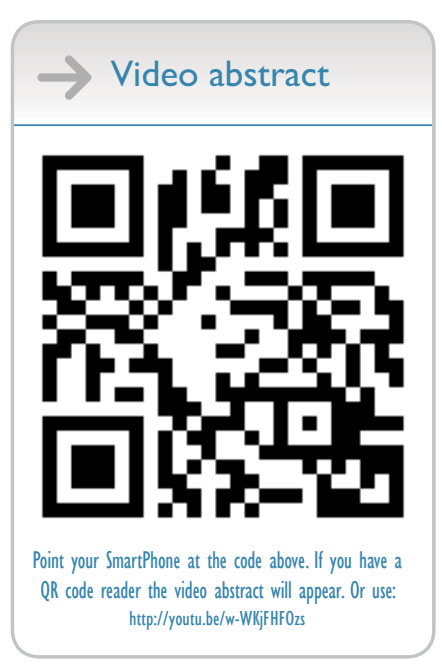

Correspondence: James A Chiarotto Department of Medicine, Scarborough and Rouge Hospital, Centenary Site, 1052863 Ellesmere Road, Scarborough, ON MIE 5E9, Canada

$\mathrm{Tel}+|4| 62848|3|$ ext 4666

Fax + I 4162817228

Email jchiarotto@bell.net
Introduction: Exercise can improve the symptoms of cancer. However, is it a cancer treatment? We tested the feasibility of group exercise for metastatic cancer patients while on chemotherapy. A biomarker for exercise efficacy in colorectal cancer (CRC), $\beta$-catenin, was tested.

Methods: Patients undergoing palliative chemotherapy were eligible for a pre-post, single-arm study comprising an indefinite, weekly group exercise intervention using strength and aerobic training. The Functional Assessment of Chronic Illness Therapy (FACIT) and Piper Fatigue Scale (PFS) questionnaires were administered, and aerobic capacity assessed using the 6-minute walk test. Selection bias, as measured by invitation rate, as well as participation, compliance, and attrition rates, was measured. CRC patients had surgical sections stained for $\beta$-catenin and correlated to survival. The statistical analysis was primarily exploratory and hypothesis generating. Results: Of the 124 eligible patients, 53 (43\%) patients were invited and 35 (28\%) patients participated. The median number of classes attended was 16 , the compliance rate was $73.1 \%$ (95\% confidence interval [CI] 67.0-79.4), and the modified attrition rate was $24 \%$. There were no injuries. No significant improvements were seen in the FACIT or PFS at 30 weeks. Aerobic capacity significantly improved at 30 weeks. Participation of CRC patients in the exercise pilot vs nonparticipation was not associated with a change in survival (hazard ratio $[\mathrm{HR}]=0.98,95 \%$ CI 0.32-2.97). For all CRC patients, strong nuclear staining for $\beta$-catenin, compared to weak, suggested a lower risk of mortality ( $\mathrm{HR}=0.54,95 \% \mathrm{CI} 0.14-1.96)$. However, CRC participants in the exercise program with weak nuclear staining for $\beta$-catenin had a trend to lower mortality (HR $=0.39,95 \%$ CI 0.025-6.1).

Conclusion: Exercise for patients with metastatic cancer receiving chemotherapy is feasible and safe. $\beta$-Catenin is a potential biomarker for exercise anticancer effect in CRC.

Keywords: strength training, aerobic training, colorectal cancer, compliance rate, attrition rate, fatigue

\section{Introduction}

The emerging field of exercise oncology is trying to determine whether exercise can impact cancer-specific outcomes. Research has already looked at how exercise can improve cancer symptoms of fatigue and physical fitness. ${ }^{1,2}$ Epidemiological evidence suggests that individuals who are active have a lower risk of developing cancer. ${ }^{3}$ Similarly, active individuals with early-stage cancer have a lower risk of cancer relapse. ${ }^{4}$ Exercise may have anticancer effects similar to standard systemic treatments ${ }^{3}$ however, this is yet to be proven in prospective randomized trials. The anticancer effect of exercise 
may be most easily seen in the metastatic setting, similar to the evaluation of investigational systemic treatments.

Exercise for patients with early stage cancer undergoing treatment is safe..$^{5}$ This pilot study evaluates the feasibility of exercising individuals with incurable metastatic malignancy receiving chemotherapy. Additionally, the proportion of such individuals invited to exercise, selection bias, attrition and compliance rates, and treatment dose intensity (DI) ${ }^{6}$ of participants was measured.

Beyond exercise's improvements in patient-related outcomes and fitness, there is now substantial interest in cancerrelated outcomes. These outcomes have significant variability among patients, and efforts are being made understand these differences. ${ }^{7}$ Genetic and molecular markers may be the predictors of these responses and help guide patient selection.

A biomarker that may predict sensitivity of colorectal cancer (CRC) to exercise is $\beta$-catenin, part of the WNT signaling pathway. ${ }^{8}$ This pathway is involved in both colorectal carcinogenesis and energy metabolism. In this study, the survival of patients with metastatic CRC participating in the exercise pilot was compared with those not participating using $\beta$-catenin nuclear staining to predict exercise effect on survival.

\section{Methods}

\section{Study design and patient selection}

A pre-post, single-arm study was designed to test the feasibility of exercising patients with incurable metastatic cancer on chemotherapy. Participants were recruited from the Chemotherapy Clinic at Rouge Valley Centenary Hospital (as of December 1, 2016, Scarborough and Rouge Hospital-Centenary Site) in Toronto, between February 2014 and August 2016. Only patients under the care of one medical oncologist (JAC) were eligible. All such patients with incurable metastatic malignancy receiving chemotherapy were candidates. There were no formal exclusion criteria. The study was approved by the Research Ethics Board at the Rouge Valley Health System. All participants provided written informed consent for this study.

Kinesiologists experienced in exercise therapy in cardiac rehabilitation supervised participants on a dedicated track with access to resistance and aerobic training machines within the Rouge Valley Centenary Hospital. Basic first-aid, CPR, and an emergency response team were available. Parking was free on the day of the group exercise.

\section{Assessments}

A kinesiologist discussed exercise history, limitations, and addressed any concerns. A 6-minute walk test determined baseline aerobic capacity. Changes were tracked every 3 months. The Functional Assessment of Chronic Illness Therapy (FACIT) and Piper Fatigue Scale (PFS) questionnaires were administered at baseline and at 30 weeks. FACIT is a quality-of-life questionnaire tested for reliability and validity in a general cancer population ${ }^{9}$ (FACT-G) with a tumor-site specific form (FACT-Specific). The PFS measures a cancer patient's fatigue tested for reliability and validity in a general cancer population. ${ }^{10}$

\section{Exercise intervention}

The intervention was a supervised, weekly, group, strength and aerobic exercise program. One 75-minute time block was allocated during the week. Two kinesiologists case managed and supervised all exercise participants. For strength training, participants used free weights or resistance bands targeting the five major muscle groups that included arms, legs, shoulders, back, and core. Five to seven total exercises were performed in each session for two sets of 10 repetitions at each group exercise session. For aerobic training, participants were encouraged to walk or cycle as a part of their aerobic training routine for up to 45 minutes. Participants were encouraged to perform their strength and aerobic exercises throughout the remainder of the week. The exercise intervention was indefinite and lasted as long as the patient wished to participate. Scheduling of the exercise sessions was just before a clinic visit or chemotherapy treatment, which continued uninterrupted according to the cancer treatment plan.

Participants submitted weekly written diaries at each exercise session to monitor home exercise. They were then given a new diary for the week as well as an exercise prescription based on their individual capacity, including aerobic and strengthening exercises.

\section{Measurements}

Demographic data and primary cancer site and chemotherapy treatment information were available for all patients treated with palliative intent by the medical oncologist during the time the study was active. Attendance for group sessions was recorded by the kinesiologist.

Prognostic factors collected at baseline for patients with metastatic CRC consisted of patient performance status measured using the Eastern Cooperative Oncology Group scale, and white blood count, absolute neutrophil count, alkaline phosphatase, number of metastatic sites, right vs left side of primary cancer, K-ras status, and number of lines of chemotherapy ${ }^{11,12}$ with previous adjuvant chemotherapy also included. 
Attrition typically looks at the rate of withdrawal from an exercise program with a fixed duration, comparing the number of participants at the beginning and conclusion. ${ }^{13}$ This pilot did not have a fixed duration of exercise participation. These were patients with incurable cancer with a chance of dying while in the pilot study. Additionally, many completed their course of palliative treatment, with no obligation to continue in the pilot study. Attrition was defined as withdrawal from the pilot earlier than 90 days before death or later than 30 days after completion of palliative chemotherapy.

The compliance rate was defined as the number of group exercise attendances divided by the number of available group exercise sessions between the first and last attended exercise sessions for each participant.

Survival for CRC patients was calculated as the time elapsed between the diagnostic biopsy and death.

Paraffin-embedded tissue blocks were collected for every patient with metastatic colon or rectal cancer treated by the medical oncologist, while the exercise pilot was active, regardless of participation in the pilot. The sample was always from a pretreatment specimen: a surgical section $(n=14)$ or biopsy $(n=3)$ for colon cancer or a biopsy for rectal cancer (Table 1). This was stained for $\beta$-catenin with normal colonic epithelial cells serving as internal controls. A team of five pathologists graded the nuclear staining as weak $(0,+1,+2)$, indicating intact regulation of the WNTCTNNB1 pathway, or strong $(+3$ or +4$)$, indicating aberrant upregulation of the WNT-CTNNB1 pathway.

Table I Patients referred

\begin{tabular}{|c|c|c|c|}
\hline Site & $\begin{array}{l}\text { Number } \\
\text { available }\end{array}$ & $\begin{array}{l}\text { Number } \\
\text { referred }\end{array}$ & Accepted \\
\hline \multicolumn{4}{|l|}{ Gastrointestinal } \\
\hline Colon & 17 & 17 & II \\
\hline Rectum & 3 & 3 & $\mathrm{I}$ \\
\hline Hepatobiliary & 7 & 1 & 0 \\
\hline Stomach & 3 & 1 & 0 \\
\hline \multicolumn{4}{|l|}{ Lung } \\
\hline Nonsmall cell & 10 & 4 & 2 \\
\hline Small cell & 6 & 1 & 0 \\
\hline Breast & 15 & II & 8 \\
\hline Gynecologic & 9 & 3 & 3 \\
\hline Prostate & 2 & 0 & 0 \\
\hline \multicolumn{4}{|l|}{ Hematologic } \\
\hline Low-grade NHL & 23 & 8 & 7 \\
\hline Multiple myeloma & 25 & 4 & 3 \\
\hline Myelodysplastic syndrome & 3 & 0 & 0 \\
\hline T-cell lymphoma & I & 0 & 0 \\
\hline
\end{tabular}

Note: Total $\mathrm{N}=124$, referred $=53$, and at least one class $=35$.

Abbreviation: NHL, non-Hodgkin lymphoma.

\section{Immunohistochemical analysis for}

\section{$\beta$-catenin}

$\beta$-Catenin is a ready-to-use/predilute antibody (catalog \# IR702; Dako/Agilent, Santa Clara, CA, USA). Dako Envision Flex+ kits (catalog \#K8002) were used for detection. The slides were cut at $3 \mu \mathrm{m}$ and dried in the oven at $60^{\circ} \mathrm{C}$ for 30 minutes, then dewaxed with xylene and hydrated, and then put into a high $\mathrm{pH}$ retrieval buffer for 20 minutes at $98^{\circ} \mathrm{C}$. The slides were rinsed with buffer and then loaded onto Dako Autostainers where they underwent the staining process (peroxidase block, $\beta$-catenin antibody [20 minutes incubation], horseradish peroxidase polymer, and diaminobenzidine substrate-chromogen). The slides were counterstained with hematoxylin manually and coverslipped for review.

\section{Data analysis}

To assess the impact of exercise on cancer treatment delivery, chemotherapy DI was determined for all patients involved in the trial with CRC and myeloma patients being compared to age- and sex-matched nonparticipants.

Demographic and clinical data were presented as mean, median, or proportion with $95 \%$ confidence intervals (CIs). Given the exploratory nature of the study, no formal sample size or power analysis was undertaken. Therefore, the most relevant outcomes from the statistical analysis were $95 \%$ CI. $P$-values should be viewed as being of secondary importance. Furthermore, the findings of this pilot study should be seen as exploratory and hypothesis generating. The paired $t$-test was used to compare changes in the 6-minute walk test relative to baseline. The association between participation in the exercise program, nuclear staining for $\beta$-catenin, and overall survival was assessed using Cox proportional hazard regression. Survival curves were generated with the method of Kaplan-Meier and compared with the log-rank test.

All of the statistical analyses were performed using Stata, release 14.0 (StataCorp LP, College Station, TX, USA).

\section{Results}

A total of 124 patients were eligible (Table 1) with a mean age of 66.2 years (range 37-94 years). The average age of patients invited to participate was 62.6 years (95\% CI 59.6-65.7), significantly different from those who were not (68.6 years) (95\% CI 66.2-70.9; $P=0.0026)$. A total of 53 patients were invited to participate; 41 patients accepted and 35 patients completed the initial assessment. No difference was seen in the invitation rate when sex, race, and tumor type were analyzed.

The medical oncologist reviewed reasons for noninvitation. The category accounting for $48 \%$ of such patients 
Table 2 Physician reasons for noninvitation/nonparticipation, $\mathrm{N}=7$ I

\begin{tabular}{|c|c|c|c|}
\hline \multicolumn{4}{|l|}{ Reasons (N/\%) } \\
\hline Cancer related & $\begin{array}{l}\text { Treatment } \\
\text { related }\end{array}$ & Social & $\begin{array}{l}\text { MD } \\
\text { related }\end{array}$ \\
\hline Elderly/frail (I5) & $\begin{array}{l}\text { Intense } \\
\text { treatment (7) }\end{array}$ & Unilingual (5) & $\begin{array}{l}\text { No clear } \\
\text { reason (8) }\end{array}$ \\
\hline $\begin{array}{l}\text { Rapid } \\
\text { deterioration (9) }\end{array}$ & Almost done (2) & Distance (2) & \\
\hline $\begin{array}{l}\text { Orthopedic/ } \\
\text { neurologic (I0) }\end{array}$ & $\begin{array}{l}\text { Await response } \\
\text { (2) }\end{array}$ & $\begin{array}{l}\text { Patient } \\
\text { declined (II) }\end{array}$ & \\
\hline Totals (N) 34 & II & 18 & 8 \\
\hline Totals (\%) 48 & 16 & 25 & II \\
\hline
\end{tabular}

Abbreviation: MD, medical doctor.

described cancer-related issues with advanced age and/or fatigue; rapid deterioration and death within the first or second cycle of treatment; and orthopedic and/or neurological issues precluding exercise participation. In $11 \%$ of cases, there were no clear reasons for noninvitation (Table 2).

The attrition rate was $24 \%$ using the previously stated definition. The rate of participation varied (Figure 1), and the median number of weekly classes attended was 16 (range 1-89). The mean compliance rate was $73.1 \%$ (95\% CI 67.0-79.4). During this study, 15 participants died, 7 participants completed their palliative systemic treatment and stopped participating, and 1 patient moved to another hospital.

Patients who died stopped participating in the exercise program at a mean of 164 days ( $95 \%$ CI $76.5-251$, median 100 days) prior to their death. Patients completing their course of palliative chemotherapy continued to participate in the exercise program a mean of 7.6 days after completion of treatment (95\% CI 73.1 days prior to ending chemotherapy to 88.3 days after completing chemotherapy).

There were no pre-to-post changes in the FACIT-General $(P=0.83)$, FACIT-Specific $(P=0.31)$, or PFS $(P=0.98)$ at 30 weeks.

Participants improved their 6-minute walk test at posttest by an average of $75 \mathrm{~m}(467 \mathrm{~m}, 95 \%$ CI $425-508 \mathrm{~m}$ vs $542 \mathrm{~m}$, 95\% CI 483-600 m, $P=0.002$ ).

For CRC patients, the average DI of participants was $96 \%$ $(\mathrm{n}=12)$, and for nonparticipants, it was significantly lower at $86 \%$ (95\% CI 75-96, $P=0.035)$. For multiple myeloma patients, participant's DI was $100 \%(\mathrm{n}=3)$, and for nonparticipants, it was $98 \%$ (95\% CI 93-104; $P=0.37$ ).

Twenty patients with metastatic CRC were treated with chemotherapy during the pilot study, with surgical sections available for 19 patients, 12 accepting the invitation and 9 taking part in the exercise pilot (Table 1). $\beta$-Catenin staining and survival analysis were undertaken for all metastatic CRC patients with available pathology specimens treated with chemotherapy during the conduct of this pilot study, regardless of participation in the exercise pilot. Fourteen of the patients died, with a median survival of 2.45 years $(95 \%$ CI 1.9-6.4). Forty-two percent of the sections stained strongly for $\beta$-catenin in the nucleus. For overall mortality, strong nuclear staining for $\beta$-catenin was associated with a lower risk of death $(\mathrm{HR}=0.54,95 \% \mathrm{CI} 0.14-1.96)$. Participation in the exercise pilot was not associated with any difference in survival (HR $=0.98,95 \%$ CI 0.32-2.97, Figure 2A). However,

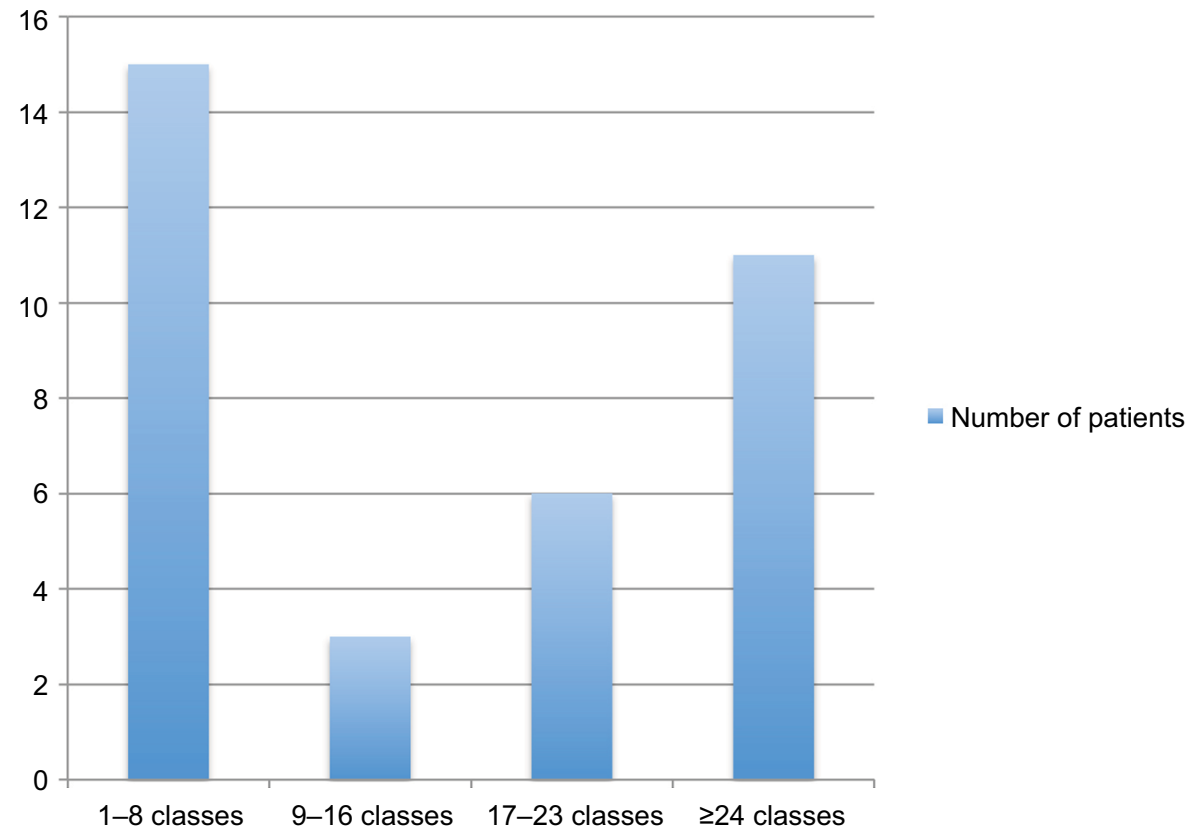

Figure I Number of weekly classes attended by study participants. 
A

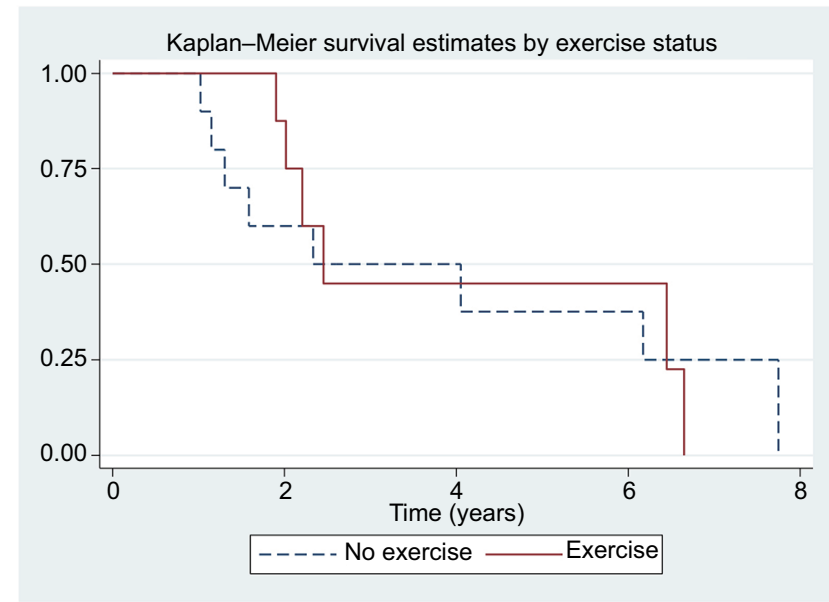

B

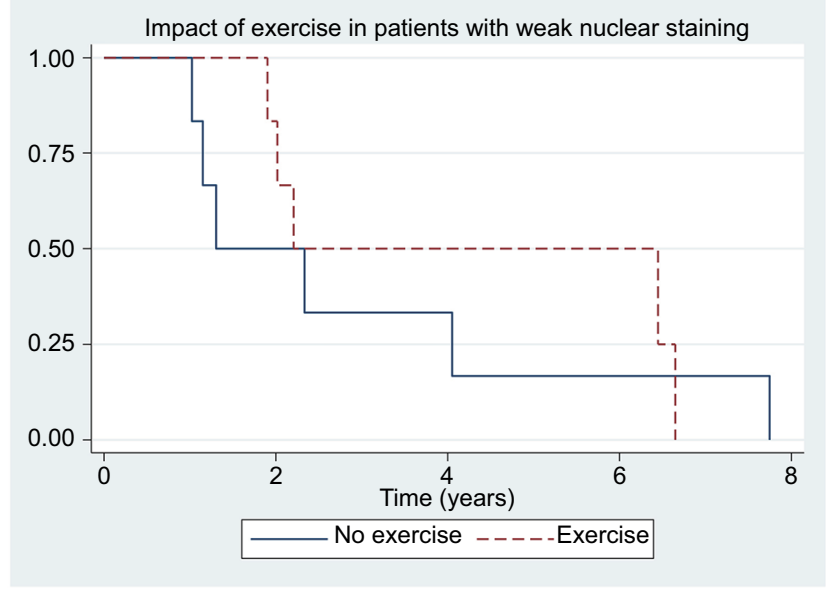

Figure 2 Kaplan-Meier survival estimates of various patients with metastatic CRC.

Notes: (A) Survival by participation in exercise program for patients with metastatic CRC. Hazard ratio (exercise, $n=9$ vs no exercise, $n=10)=0.98,95 \%$ Cl $0.32-2.97$. (B) Survival by participation in exercise program for patients with weak $\beta$-catenin nuclear staining and metastatic CRC. Hazard ratio (exercise, $n=7$ vs no exercise, $n=6$ ) $=0.39$, 95\% Cl 0.025-6.I.

Abbreviations: $\mathrm{CRC}$, colorectal cancer; $\mathrm{Cl}$, confidence interval.

participants in the exercise pilot who had weak nuclear staining for $\beta$-catenin had a lower risk of mortality $(H R=0.39$, 95\% CI 0.025-6.1, Figure 2B).

\section{Discussion}

Few studies looking at the benefits of exercise in patients with advanced cancer have been published. ${ }^{14}$ Lowe et a ${ }^{15}$ reviewed six studies of physical activity and palliative cancer patients, all pilots, involving a total of 84 patients. Subsequent studies looking at exercise and late-stage cancer patients included between 21 and 65 individuals, ${ }^{13,16-18}$ receiving strictly palliative chemotherapy included in heterogeneous groups of patients. This pilot examined 35 patients all with metastatic, incurable malignancy on cytotoxic systemic treatment.

To our knowledge, this is the first exercise pilot using metastatic cancer patients receiving only palliative chemotherapy in a general oncology practice. This pilot did not document any adverse effects, similar to studies with the same patient population. ${ }^{16-18}$ Two other studies exercising individuals with only multiple myeloma ${ }^{19}$ and metastatic breast cancer ${ }^{13}$ reported no injuries. Existing recommendations state that it is safe for cancer survivors to exercise..$^{5,20}$ Our observations are consistent with observations that exercising patients with metastatic disease is safe. ${ }^{19}$ Currently, no exercise recommendation exists for this group of patients.

One goal was to determine the participation rate of metastatic cancer patients on chemotherapy in an exercise program: $28 \%(35 / 124)$ of potential candidates. This agrees with many prospective trials, where less than one-third of eligible patients participate. ${ }^{4}$ This pilot's potential maximum (according to Table 2, given perceived cancer-related issues precluding participation) includes an additional 37 patients for a total of $72(35+37)$ or $58 \%(72 / 124)$. The highest participation rate in the literature was $82 \%$ using inpatients in a 4-week program. ${ }^{21}$ Eleven (8.9\%) patients declined the invitation. This pilot study was designed for maximum convenience of the patient: exercise and chemotherapy in the same hospital, clinical appointment shortly after the exercise session, and paid parking on exercise day.

We wanted to understand selection bias as opposed to informal exclusion criteria. While work has been done to assess patient-related factors, ${ }^{4,15,22}$ health care team biases ${ }^{4}$ have not been studied as extensively. A palliative chemotherapy candidate may not be an exercise candidate for valid medical reasons. While some patients can successfully exercise, others may fall into a category where a judgment is required by the medical team. A common belief is that cancer patients should rest rather than exercise, especially individuals with metastatic disease. In this pilot study, in the judgment of this particular medical oncologist (JAC), $48 \%$ of patients not invited were incapable of effectively taking part in the exercise program. This may be related to the aforementioned thinking. The remaining $52 \%$ are biases related to treatment or social factors (Table 2). Our study found that younger patients are more likely to be asked to participate. Further clarification of health care team bias or informal exclusion criteria is needed.

Regarding acceptable tolerability and safety, a study attrition rate of $\leq 20 \%$ and an exercise compliance rate of $\geq 70 \%$ have been suggested as acceptable. ${ }^{23}$ In this pilot study, the 
attrition rate was $24 \%$ using the previously stated definition. This compares to an attrition rate of $41 \%$ in a similar population of incurable metastatic patients in a 6-month program ${ }^{19}$ and $34 \%$ in women with metastatic breast cancer in a 16 -week program, $45 \%$ of whom were receiving chemotherapy. ${ }^{13}$ Our study determined that patients stopped participating in the exercise program a median of 100 days prior to death and, for those patients completing palliative chemotherapy, continued to participate in the exercise program for an average of 7.6 days beyond their treatment. The compliance rate for class attendance in our indefinite study was $73.1 \%$ (median $73.9 \%$ ). A compliance rate of $99 \%$ has been achieved in a 12-week program in the adjuvant setting. ${ }^{24}$ Therefore, the attrition rate using a modified definition and the compliance rate are comparable with what is acceptable for therapeutic intervention.

In this study, palliative chemotherapy treatment was not interrupted by the exercise schedule as indicated by preserved DI. Similar findings were seen in the setting of exercise and adjuvant chemotherapy for breast cancer. ${ }^{25}$

Our study did not show an improvement in the quality of life or reduction in fatigue with exercise, similar to other studies with this patient population. ${ }^{13,17,18}$ The improvement in physical function with exercise that we found has been reported in this patient population ${ }^{17,18}$ but not universally. ${ }^{13}$

How exercise might improve cancer survival is not clear. Medical oncology has moved toward targeted therapy, and exercise oncology must, similarly, determine which patients benefit from exercise as epidemiological data suggest differences with tumor subtype. ${ }^{23} \beta$-Catenin is a candidate biomarker for exercise effect in CRC. ${ }^{8}$ In surgical and biopsy sections from our metastatic CRC patients, $42 \%$ stained strongly for $\beta$-catenin in the nucleus where Morikawa et al found $46 \%$ for early-stage CRC patients. Morikawa et al showed that strong nuclear staining for $\beta$-catenin was associated with a significant reduction in mortality $(\mathrm{HR}=0.56,95 \%$ CI $0.35-0.90)$ in patients with early-stage CRC but only among patients with a body mass index (BMI) of $\geq 30 \mathrm{~kg} / \mathrm{m}^{2}$. In our study, strong nuclear staining was associated with a nonsignificant reduction in the risk of death for all patients $(\mathrm{HR}=0.54,95 \% \mathrm{CI}$ 0.14-1.96), but we were unable to confirm Morikawa et al's finding with respect to BMI given our smaller data set.

Exercise did not affect survival ( $\mathrm{HR}=0.98,95 \% \mathrm{CI} 0.32-$ 2.97) of patients with metastatic CRC in our study (Figure 2A). However, metastatic CRC patients with weak nuclear staining for $\beta$-catenin participating in our exercise program had a nonsignificant mortality reduction $(\mathrm{HR}=0.39,95 \%$ CI $0.025-6.1$ ) (Figure 2B). This is similar to the mortality reduction described by Morikawa et al for patients with weak nuclear staining, physical activity, and early-stage colon cancer (HR 0.33, 95\% CI 0.42-1.09). These results support an intact regulation of the WNT-CTNNB1 pathway being required for exercise to have a beneficial effect on cancer progression, with weak nuclear staining for $\beta$-catenin being a potential biomarker. These hypotheses warrant further investigation.

Limitations of this study include the lack of measurement of physical activity including during home exercise where self-reporting can be subjective and lack reliability. Given that there are thresholds of physical activity needed to induce improvements in cancer-specific endpoints, ${ }^{3}$ this study cannot determine what that might be. Individualized prescriptions that use tenets of exercise treatment such as peak rate of oxygen consumption (peak $\mathrm{VO}_{2}$ ) and progressive overload have not been used the in the field of exercise oncology ${ }^{26}$ and were not used in this pilot. The invitation rate was based on the judgment of the medical oncologist and may differ depending on the health care professional. The $\beta$-catenin survival study suffers from small sample size, lack of randomization, difficulty in controlling for known prognostic factors in metastatic CRC, and lack of information with respect to activity of nonexercising control patients.

Future work will include a prospective trial looking at referral bias and the development of systematic exclusion criteria, a more careful measurement of physical activity to understand dosing of exercise and possible threshold effects, the use of training effect, a prospective trial of exercise, and $\beta$-catenin staining as the biomarker.

\section{Conclusion}

This study has tried to address several of the many unsolved issues regarding the effects of exercise in metastatic cancer patients. ${ }^{4}$ The first is the issue surrounding precision of the data generated with the inclusion of descriptions of the exercise program, and participation, attrition, and compliance rates. We also looked into reasons for referral bias to an exercise program and established a maximum participation rate for metastatic patients. The lack of this sort of data has been responsible for the wide variation in outcomes in previously published literature. ${ }^{4}$ We have added to the experience that exercise is safe in the metastatic population and that chemotherapy DI is not altered. We offered an exercise program with maximum convenience and safety, available as long as the patient wished to participate. We also looked into a potential biomarker for the efficacy of exercise. This will become especially important as the intracellular signaling 
pathways involved with exercise and cancer are elucidated, with $\beta$-catenin as one such candidate.

\section{Acknowledgments}

The authors thank our patients for their participation; Kathryn Leeder for her inspiration, Pamela West for her editorial support; Dr Daniel Santa Mina and Dr Paul Oh for advice and guidance; and Dr Soran Abdulkarim, Dr Dina El-Sahrigy, Dr Allan Hunt, Dr Helena Meskel, Dr Bassem Moussa, and Dr Ron Stead for their work on the $\beta$-catenin staining. The authors alone are responsible for the content and writing of the article.

\section{Disclosure}

Dr George Dranitsaris owns and is a consultant for Augmentium Pharma Consulting, Inc. The authors report no other conflicts of interest in this work.

\section{References}

1. Cramp F, Daniel J. Exercise for the management of cancer-related fatigue in adults. Cochrane Database Syst Rev. 2010;11:CD006145.

2. Markes M, Brokow T, Resch K. Exercise for women receiving adjuvant therapy for breast cancer. Cochrane Database Syst Rev. 2009; 4:CD005001.

3. Betof AS, Dewhirst MW, Jones LW. Effects and potential mechanisms of exercise training on cancer progression: a translational perspective. Brain Behav Immun. 2013;30(Suppl):S75-S87.

4. Bouillet T, Bigard X, Brami C, et al. Role of physical activity and sport in oncology scientific commission of the National Federation Sport and Cancer CAMI. Crit Rev Oncol Hematol. 2015;94(1):74-86.

5. Schmitz KH, Courneya KS, Matthews C, et al; American College of Sports Medicine. American College of Sports Medicine roundtable on exercise guidelines for cancer survivors. Med Sci Sports Exerc. 2010;42(7):1409-1426.

6. Balducci L, Mo M, Abella E, Saven A. Retrospective analysis of relative dose intensity in patients with non-Hodgkins lymphoma receiving CHOP-based chemotherapy and pegfilgrastim. Am J Clin Oncol. 2014;37(6):603-610.

7. Friedenreich CM, Neilson HK, Farris MS, Courneya KS. Physical activity and cancer outcomes: a precision medicine approach. Clin Cancer Res. 2016;22(19):4766-4775.

8. Morikawa T, Kuchiba A, Yamauchi M, et al. Association of CTNNBI (B-Catenin)alterations, body mass index, and physical activity with survival in patients with colorectal cancer. JAMA. 2011;305(16):1685-1694.

9. Cella DF, Tulsky DS, Gray G, et al. The functional assessment of cancer therapy scale: development and validation of the general measure. JClin Oncol. 1993;11(3):570-579.
10. Mota DD, Pimenta CA, Piper B. Fatigue in Brazilian cancer patients, caregivers, and nursing students: a psychometric validation study of the Piper Fatigue Scale-Revised. Support Care Cancer. 2009;17(6):645-652.

11. Kohne C, Cunningham D, Di Costanzo F, et al. Clinical determinants of survival in patients with 5-fluorouracil based treatment for metastatic colorectal cancer: results of a multivariate analysis of 3825 patients. Ann Oncol. 2002;13(2):308-317.

12. Foltran L, De Maglio G, Pella N, et al. Prognostic role of KRAS, NRAS, BRAF and PIK3CA mutations in advanced colorectal cancer. Future Oncol. 2015;11(4):629-640.

13. Ligibel JA, Giobbie-Hurder A, Shockro L, et al. Randomized trial of physical activity intervention in women with metastatic breast cancer. Cancer. 2016;122(8):1169-1177.

14. Chasen M, Bhargava R, MacDonald N. Rehabilitation for patients with advanced cancer. CMAJ. 2014;186(14):1071-1075.

15. Lowe SS, Wantanabe SM, Courneya KS. Physical activity as supportive care intervention in palliative care: a systematic review. J Support Oncol. 2009;7(1):27-34.

16. Adamsen L, Quist M, Midtgaard J, et al. The effect of a multidimensional exercise intervention on physical capacity, well-being and quality of life in cancer patients undergoing chemotherapy. Support Care Cancer. 2006;14(2):116-127.

17. Adamsen L, Quist M, Andersen C, et al. Effect of a multimodal high intensity exercise intervention in cancer patients undergoing chemotherapy: randomised controlled trial. BMJ. 2009;339:b3410.

18. Oldervoll LM, Loge JH, Lydersen S, et al. Physical exercise for cancer patients with advanced disease: a randomized controlled trial. Oncologist. 2011;16(11):1649-1657.

19. Coleman EA, Coon S, Hall-Barrow J, Richards K, Gaylor D, Steward B. Feasibility of exercise during treatment for multiple myeloma. Cancer Nurs. 2003;26(5):410-419.

20. Henningsen KH, Desomer A, Hanssens S, Vlayen J. Supportive Treatment for Cancer Part 1: Exercise Treatment. Brussels, Belgium: Belgian Health Care Knowledge Centre (KCE); 2012.

21. Bertheussen GF, Kaasa S, Hokstad S, et al. Feasibility and changes in symptoms and functioning following inpatient cancer rehabilitation. Acta Oncol. 2012;51(8):1070-1080.

22. Kartolo A, Cheng S, Petrella T. Motivation and preferences of exercise programmes in patients with operable metastatic lung cancer: a needs assessment. Support Care Cancer. 2016;24(1):129-137.

23. Jones LW. Precision oncology framework for investigation of exercise as treatment for cancer. J Clin Oncol. 2015;33(35):4134-4137.

24. Rogers L, Hopkins-Price P, Vicari S, et al. A randomized trial to increase physical activity in breast cancer survivors. Med Sci Sports Exerc. 2009;41(4):935-946.

25. Courneya KS, Segal RJ, Mackey JR, et al. Effects of aerobic and resistance exercise in breast cancer patients receiving adjuvant chemotherapy: a multicenter randomized controlled trial. J Clin Oncol. 2007;25(28):4396-4404.

26. Sasso JP, Eves ND, Christensen JF, Koelwyn GJ, Scott J, Jones LW. A framework for prescription in exercise-oncology research. J Cachexia Sarcopenia Muscle. 2015;6(2):115-124.
Cancer Management and Research

\section{Publish your work in this journal}

Cancer Management and Research is an international, peer-reviewed open access journal focusing on cancer research and the optimal use of preventative and integrated treatment interventions to achieve improved outcomes, enhanced survival and quality of life for the cancer patient. The manuscript management system is completely online and includes

\section{Dovepress}

a very quick and fair peer-review system, which is all easy to use. Visit http://www.dovepress.com/testimonials.php to read real quotes from published authors. 